\title{
On the definition of schemes for computing leading order isospin breaking corrections
}

\author{
A. Bussone ${ }^{* a, b}$, M. Della Morte ${ }^{c}$, T. Janowski ${ }^{d}$, A. Walker-Loud ${ }^{e, f}$ \\ ${ }^{a}$ Department of Theoretical Physics, Universidad Autónoma de Madrid, E-28049 Madrid, Spain \\ ${ }^{b}$ Instituto de Física Teórica UAM-CSIC, c/ Nicolás Cabrera 13-15, Universidad Autónoma de \\ Madrid, E-28049 Madrid, Spain \\ ${ }^{c} C P^{3}$-Origins, University of Southern Denmark, Campusvej 55, 5230 Odense, Denmark \\ ${ }^{d}$ School of Physics and Astronomy, University of Edinburgh, Edinburgh EH9 3JZ, UK \\ ${ }^{e}$ Nuclear Science Division, Lawrence Berkeley National Laboratory, Berkeley, CA 94720, USA \\ ${ }^{f}$ Physics Division, Lawrence Livermore National Laboratory, Livermore, CA 94550, USA \\ E-mail:andrea.bussone@uam.es, dellamor@cp-origins.net, \\ t.janowski@ed.ac.uk, awalker-loudelbl.gov
}

\begin{abstract}
We discuss an alternate scheme, or a 'line of constant physics', which can be used when computing isospin breaking corrections to hadronic quantities. We show that within a certain class of schemes one can separate the electromagnetic corrections from the strong isospin breaking corrections at leading order, meaning that within this class scheme-ambiguities are higher order effects.
\end{abstract}

The 36th Annual International Symposium on Lattice Field Theory - LATTICE2018

22-28 July, 2018

Michigan State University, East Lansing, Michigan, USA.

\footnotetext{
${ }^{*}$ Speaker.
} 


\section{Introduction}

Lattice QCD has reached in many instances such a level of precision that the breaking of isospin symmetry has become the main source of uncertainty (see, for example [1] and [2]). This breaking has two origins; one is due to the difference in the electric charge of the light quarks (typically indicated as "EM" or "QED" breaking) and the other is due to their difference in mass (typically indicated as "strong ispsopin" breaking). Isospin breaking from these two sources is intimately coupled as the electromagnetic interactions renormalize the quark mass operators, which in turn serve as counter-terms to ultra-violet divergences arising from radiative QED corrections. Therefore, assessing the amount isospin breaking arising from QED or strong sources, and the underlying Lagrangian parameters, is necessarily renormalization scheme dependent.

In order to obtain results with accuracies beyond the percent level, as needed for example for the hadronic contribution to the anomalous magnetic moment of the muon, lattice calculations have to depart from the isospin symmetric limit. One possible approach consists in "simply" simulating QED+QCD with $N_{f}$ quarks of different masses. Such a theory has $N_{f}+2$ parameters, and assuming it gives an accurate description of Nature (at low energies, perhaps), one can fix those parameters by choosing $N_{f}+2$ "reasonably independent" experimental inputs. Upon choosing a renormalization scheme, these bare parameters are then converted to renormalized ones. Any other choice of $N_{f}+2$ "reasonably independent" experimental inputs would produce the same renormalized parameters, again assuming that QED+QCD with $N_{f}$ flavors gives an accurate description of Nature (and barring accidental degeneracies). In this approach there is no "scheme dependence" (meaning dependence on the choice of quantities used to fix the $N_{f}+2$ paramaters) in the results.

However, for different reasons, one is often interested in estimating the size of isospin symmetry breaking corrections, by imagining a Taylor expansion of observables around the isospin symmetric point. For example, the primordial amount of ${ }^{4} \mathrm{He}$ produced in Big-Bang-Nucleosynthesis is very sensitive to isospin breaking [3]. Quantitatively assessing the dependence from the two sources of isospin breaking allows for strong constraints to be placed upon the possible timevariation of this fundamental constant. We elaborate more on the Taylor expansion mentioned above in this proceedings contribution, however, it is clear that in comparing an "isospin symmetric world" to one which is not, one has to specify what is kept fixed in the comparison and that produces a scheme ambiguity on which we collect a number of remarks in the following. We discuss in some detail, a class of schemes in which the scheme-ambiguities are second order in isospin breaking, allowing for a meaningful separation of these effects to a precision that is sufficient for current and projected lattice QCD applications.

\section{Setting up the expansion}

We consider a quantity $\mathscr{O}$ that we want to compute including QCD + IB (isospin breaking) corrections to leading order. In order to setup an expansion (for the IB contributions), we need to write its dependence on "independent" parameters. One of the subtleties is related to $\delta m=m_{d}-m_{u}$ as that is in principle a function of $\alpha$ (here being the EM coupling). 
We work in the simpler setup of $N_{f}=2$, where the main issues are however already present, and write $\mathscr{O}$ as a function of renormalized parameters (neglecting $O(\alpha)$ corrections to $\alpha_{\text {strong }}{ }^{1}$

$$
\mathscr{O}=\mathscr{O}\left(\left(m_{d}-m_{u}\right)_{R}(\alpha),\left(m_{d}+m_{u}\right)_{R}(\alpha), \alpha\right)
$$

with, say, $R=\overline{\mathrm{MS}}$ at $2 \mathrm{GeV}$. The arguments are clearly not independent, so, let us first of all fix $\left(m_{d}+m_{u}\right)_{R}(\alpha)=\left(m_{d}+m_{u}\right)_{R, p h y s}=\left(m_{d}+m_{u}\right)_{P D G} \approx 7 \mathrm{MeV}$ [5] for all values of $\alpha$. That makes the parameter $\alpha$-independent by construction. In order to achieve that, one can fix the sum of PCAC quark masses, which requires computing $O(\alpha)$ corrections to renormalization factors as $Z_{A}$ and $Z_{P}$.

An alternative option is to keep $m_{\pi^{0}}^{2}$ fixed. In $\chi$ PT the EM corrections to $m_{\pi^{0}}^{2}$ are chirally suppressed and extremely small, at the $0.2 \%$ level at NLO [6,7], or practically speaking, numerically second order in IB. This follows from the observation that $\delta m \sim m_{d}+m_{u}$. Strong IB breaking corrections to $m_{\pi^{0}}^{2}$ start at $O\left(m_{d}-m_{u}\right)^{2}$, therefore keeping $\left(m_{d}+m_{u}\right)_{R}$ fixed is equivalent, at the order we are working, to keep $m_{\pi^{0}}^{2}$ fixed at its (physical) value as we change $\alpha$. Notice however that the neutral pion correlator outside the isospin symmetric limit receives quark-disconnected contributions (also chirally suppressed, see [8]), the $\pi^{0}$ mixes with the $\eta$-like particles and beyond the electroquenched approximation it decays into two photons (although the width is very narrow)

Now one argument of $\mathscr{O}$ is fixed through one of the options above, and to remind us we write

$$
\mathscr{O}=\mathscr{O}\left(\left(m_{d}-m_{u}\right)_{R}(\alpha), 7 \mathrm{MeV}, \alpha\right)
$$

To proceed further we need the function $\left(m_{d}-m_{u}\right)_{R}(\alpha)$, i.e., the quark mass splitting as a function of $\alpha$. That however is not uniquely defined, as we need to specify what we keep constant (on top of $m_{\pi^{0}}^{2}$ ) to tune the masses as we change $\alpha$. We will look at two examples.

In the first let us say we keep fixed the neutron-proton splitting to its physical value. Then, by looking at Fig. 3 of [9], qualitatively, one gets something like the red dashed line in Fig. 1. So a decrease of about $30 \%$ at $\alpha=0$ with respect to the value at $\alpha=1 / 137$.

One can also use a different condition, perhaps yielding a smaller dependence of $\left(m_{d}-m_{u}\right)_{R}$ on $\alpha$. For example, as suggested in [9], one can keep the splitting between $\Sigma^{+}$and $\Sigma^{-}$fixed to its physical value ${ }^{2}$. Since the two baryons have the same charge (in absolute value), the EM corrections to the masses are the same at leading order in $\alpha$ (neglecting structure-dependent contributions) and the splitting is due to the $m_{d}-m_{u}$ difference only (see also [10] for a dispersive estimate). One could therefore expect a dependence as depicted by the blue dot-dashed line in Fig. 1; a much weaker one (starting at $O\left(\alpha^{2}\right)$ ) compared to the previous case.

There is no inconsistency here. The only requirement for all choices is that at the physical value of $\alpha$ all conditions give the same physical splitting ${ }^{3}$, but for $\alpha \neq 1 / 137$ one needs to specify

\footnotetext{
${ }^{1}$ Those can be absorbed into a change in the lattice spacing. One can fix the relative lattice spacing by computing $r_{0} / a$, as done in [4], or any other "gluonic" quantity (e.g., $\left.t_{0}\right)$. In the electroquenched approximation that is independent from $\alpha$ to all orders, as there is no direct coupling between photons and gluons. Beyond the electroquenched approximation one should extend the definition of the scheme by keeping fixed a quantity which depends on $\alpha_{s}$ only up to quadratic corrections in $\alpha$ and $m_{d}-m_{u}$.

${ }^{2}$ We remind the reader, $\Sigma^{+}$is a $u u s$ baryon and $\Sigma^{-}$a $d d s$ one. We are assuming here that the (valence) strange quark mass has been fixed through an hadronic condition, for example by requiring the combination $m_{K^{0}}^{2}+m_{K^{+}}^{2}$ to take its physical value.

${ }^{3}$ Strictly speaking that would happen only for the six-flavor theory with physical masses, in the infinite volume ...
} 


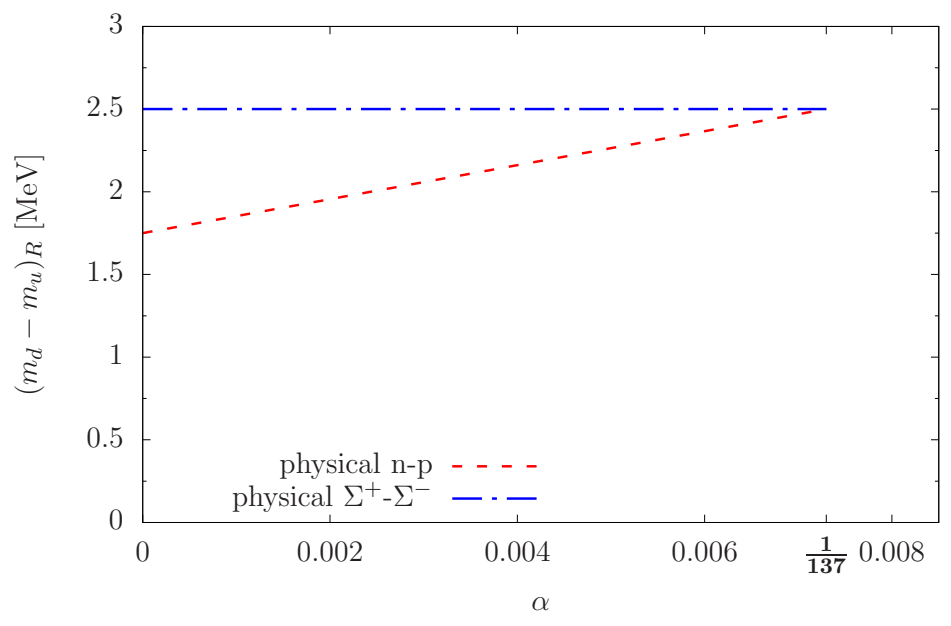

Figure 1: Red dashed: qualitative behaviour of $\left(m_{d}-m_{u}\right)_{R}(\alpha)$ as "guessed" from Fig. 3 in [9] by requiring the neutron proton splitting to stay constant. $2.5 \mathrm{MeV}$ is roughly the physical (PDG or FLAG) value for the light quark mass difference. Blue dot-dashed: Qualitative behaviour of $\left(m_{d}-m_{u}\right)_{R}(\alpha)$ by requiring the $\Sigma^{+}-\Sigma^{-}$splitting to stay constant.

what is meant by a world where $\alpha$ differs from its physical value, since there are other parameters to be fixed (in this case the mass splitting).

So, some definitions of the function $\left(m_{d}-m_{u}\right)_{R}(\alpha)$ may be better than others in some respect, but in prnciple they are all theoretically good. Since we will be interested in $\left(m_{d}-m_{u}\right)_{R}(0)$, it makes sense to ask how much that changes (parameterically) for two different definitions of the world with $\alpha \neq 1 / 137$.

Defining $\Delta_{i} m(\alpha)=\left.\left(m_{d}-m_{u}\right)_{R}(\alpha)\right|_{\text {world } i}$, and dropping the index $R$ from now on (all masses should be understood as renormalized), the requirement we just discussed implies

$$
\Delta_{1} m(\alpha=1 / 137)=\Delta_{2} m(\alpha=1 / 137)=\Delta m_{p h y s},
$$

and, by linearizing the dependence around $\alpha=1 / 137$

$$
\Delta_{i} m(\alpha)=\Delta m_{p h y s}+\left(\alpha-\frac{1}{137}\right) * c_{i}
$$

This linear hypothesis alone would give $\Delta_{1} m(0)-\Delta_{2} m(0)=O\left(\alpha_{\text {phys }}\right)$, which would translate into an ambiguity of $O(\alpha)$ once inserted in the expansion of the quantity $\mathscr{O}$, as we shall see. We need a stronger bound and for that we use the fact that in renormalized perturbation theory the EM corrections to the mass of the quarks are multiplicative as a consequence of residual chiral symmetries (axial and vector):

$$
m_{u, i}(\alpha)=m_{u, i}(0) Z_{u, i}(\alpha), \quad \text { and } \quad m_{d, i}(\alpha)=m_{d, i}(0) Z_{d, i}(\alpha)
$$

with $Z_{X, i}(\alpha)=1+C_{X, i} \alpha+\cdots$. The relation above is among renormalized quantities; in particular the mass on the r.h.s. is the renormalized mass in QCD and the coefficient $Z_{X}^{i}(\alpha)$ is expanded in powers of the renormalized EM coupling. One can therefore imagine having used a regularization preserving (part of) the chiral symmetry, where indeed quark masses renormalize multiplicatively 
even at finite lattice spacing. Notice also that the chosen "scheme" preserves the fact that the QED corrections to the quark masses are multiplicative. In fact if one were to set the neutral pion mass to zero at $\alpha=0$, which would enforce vanishing light quark masses, and then keep the same condition for all values of $\alpha$, the light quarks would remain massless (at least to the order in the EM coupling that we are considering).

The splitting now reads

$$
\begin{aligned}
\Delta_{i} m(\alpha) & =\Delta_{i} m(0) Z_{d, i}(\alpha)+\left(Z_{d, i}(\alpha)-Z_{u, i}(\alpha)\right) m_{u, i}(0) \\
& =\Delta_{i} m(0)\left(1+C_{d, i} \alpha\right)+C_{(d-u), i} \alpha m_{u, i}(0)
\end{aligned}
$$

For $\alpha=1 / 137=\alpha_{\text {phys }}$, through eq. 2.3, we obtain

$$
\begin{aligned}
& \Delta_{1} m(0)\left(1+C_{d, 1} \alpha_{p h y s}\right)+C_{(d-u), 1} \alpha_{p h y s} m_{u, 1}(0)= \\
& \Delta_{2} m(0)\left(1+C_{d, 2} \alpha_{p h y s}\right)+C_{(d-u), 2} \alpha_{p h y s} m_{u, 2}(0),
\end{aligned}
$$

which we rewrite as

$$
\begin{aligned}
\Delta_{1} m(0)-\Delta_{2} m(0)= & \alpha_{p h y s}\left(C_{d, 2} \Delta_{2} m(0)-C_{d, 1} \Delta_{1} m(0)\right)+, \\
& \alpha_{p h y s}\left(C_{(d-u), 2} m_{u, 2}(0)-C_{(d-u), 1} m_{u, 1}(0)\right) .
\end{aligned}
$$

That shows that the difference in $\Delta m$ in the two worlds is $\mathrm{O}(\alpha)$ at least (we knew that already). Similarly, by requiring the up-quark masses to be the same at $\alpha=1 / 137$, starting from eq. 2.5 , one concludes that the difference in the $m_{u, i}(0)$ is also $O(\alpha)$ at least. The previous equation can then be cast in the form

$$
\Delta_{1} m(0)-\Delta_{2} m(0)=O\left(\alpha^{2}\right)+O(\alpha \delta m)+O\left(\alpha m_{u}(0)\right) .
$$

Now, using the fact that, numerically, $\delta m \simeq m_{u}$ ( $m_{d}$ and $m_{u}$ differ roughly by a factor 2$)$, one obtains

$$
\Delta_{1} m(0)-\Delta_{2} m(0)=O\left(\alpha^{2}\right)+O(\alpha \delta m)
$$

which means that two different definitions of the $\alpha$-dependence of the up-down quark mass splitting provide values for $\Delta m(0)$ that differ by higher orders in the IB-corrections.

Coming back to the expansion of the observable $\mathscr{O}$, the conclusion is that one can either use $\Delta_{1} m$ or $\Delta_{2} m$, as long as one is interested in the leading order corrections in both $\alpha$ and $\Delta m$. We can now indeed rewrite $\mathscr{O}$ in terms of completely independent variables by using $\Delta_{1} m$ as first argument:

$$
\mathscr{O}=\mathscr{O}\left(\Delta_{1} m(0), 7 \mathrm{MeV}\left(\text { or } m_{\pi^{0}}^{2} \text { fixed to its physical value }\right), \alpha\right)
$$

which can be expanded as

$$
\begin{aligned}
\mathscr{O} & =\mathscr{O}(0,7 \mathrm{MeV}, 0)+\left.\alpha_{\text {phys }} \frac{\partial \mathscr{O}(0,7 \mathrm{MeV}, \alpha)}{\partial \alpha}\right|_{\alpha=0}+ \\
& +\left.\Delta_{1} m(0) \frac{\partial \mathscr{O}(\Delta m, 7 \mathrm{MeV}, 0)}{\partial \Delta m}\right|_{\Delta m=0}+O\left(\alpha^{2}\right)+O(\alpha \delta m)
\end{aligned}
$$


The first term should be computed in pure QCD with degenerate up and down quarks (with masses summing up to $7 \mathrm{MeV}$ or $m_{\pi^{0}}=m_{\pi^{0}, \text { phys }}$ ). For the second one needs to simulate QCD+QED tuning the bare masses for the up and down quarks, such that at each value of $\alpha$ the renormalized masses are the same. Again the sum of the quark masses must be kept fixed for all values of $\alpha$. There is an ambiguity here in the way the renormalized masses of the up and down quark are fixed to be degenerate at $\alpha \neq 0$. If the renormalization factors are known including $O(\alpha)$ corrections, one can tune the bare masses as described above. Otherwise one can adjust them such that the $\Sigma^{+}$and the $\Sigma^{-}$are degenerate, or, following [8], one can require the (squared) effective masses extracted from the connected diagrams in the two-point functions of the pseudoscalar density made either of up-quark fields only or of down-quark field only to be the same. In both cases one is requiring a quantity proportional to the mass splitting up to corrections quadratic in the IB parameters to vanish ${ }^{4}$. It is easy to see then, that the ambiguity on the second term on the r.h.s of eq. 2.12 is also quadratic in the IB parameters.

Finally, the third term can be computed in QCD with non-degenerate up and down quarks (and the by now usual requirement on the sum of the masses). The ambiguity here comes from the definition of $\Delta m$, but as we have argued in this contribution such ambiguity is second order in IB-corrections.

In conclusion, assuming all derivatives to be of $O(1)$, we see that all the ambiguities in the leading order expansion are of higher order and in particular we see that using $\Delta_{2} m(0)$ instead of $\Delta_{1} m(0)$ would change the result by $O\left(\alpha^{2}\right)$ and $O(\alpha \delta m)$, which we are anyhow neglecting. Let us also remark that since $\Delta_{1} m(0)$ differs by about $30 \%$ from the physical splitting (as one can infer from [9]), as long as we are interested in IB corrections with an accuracy of that order (which is reasonable), we could use the physical, PDG (or FLAG), $m_{d}-m_{u}$ value in eq. 2.12 instead of some $\Delta_{i} m(0)$. Notice also that all quantities in that equation are renormalized and the constraints are defined through renormalized quantities, so in principle each term can be independetly computed and extrapolated to the continuum and infinite volume limit.

\section{Summary and conclusions}

We have shown that in separating strong from EM isospin breaking effects at leading order, a rather natural class of schemes exists such that all ambiguities are of next-to-leading order. What charcterizes the schemes are the following conditions:

i) Throughout the different computations, one keeps fixed a quantity that depends on $m_{u}+m_{d}$ up to quadratic IB corrections (e.g. $m_{\pi^{0}}^{2}$ ), and in principle a quantity which at the same order only depends on $\alpha_{\text {strong }}$ (for setting the scale). For the latter, in the electroquenched approximation one can use $r_{0}$ extrapolated to the chiral limit.

ii) The condition fixing the mass splitting for $\alpha \neq 0$ should be smooth in $\alpha$. In particular we have used " $\alpha$-independent" conditions. The requirement that all such conditions provide the same splitting for $\alpha=1 / 137$, follows from the renormalizability of the theory, as discussed in the Introduction.

\footnotetext{
${ }^{4}$ For the condition used in [8], the corrections are actually $O(\alpha)$, but they are chirally suppressed.
} 
iii) The mass-symmetric point at $\alpha \neq 0$ is defined through a quantity which is proportional to the mass splitting and has no $O(\alpha)$ corrections, rather $O(\alpha \delta m)$ or $O\left(\alpha m_{\ell}\right)$.

In discussing these schemes and their ambiguities, we have often exchanged $O\left(m_{u}\right)$ or $O\left(m_{d}\right)$ with $O(\delta m)$, e.g, when neglecting the chirally suppressed $O(\alpha)$ corrections to $m_{\pi^{0}}^{2}$ or in point iii) above. While this seems reasonable close to the physical point, it is not clear to us how reliable that procedure is when working with pions with masses between 200 and $400 \mathrm{MeV}$, as it is often the case in lattice computations. Exploring such effects is left for future studies.

\section{Acknowledgments}

We acknowledge insightful conversations with C. Sachrajda concerning "hadronic" schemes for determining QED and strong isospin breaking corrections. See also the presentation at Lattice 2018 [11]. A.B. acknowledges support through the Spanish MINECO project FPA2015-68541-P, the Centro de Excelencia Severo Ochoa Programme SEV-2016-0597 and the Ramón y Cajal Programme RYC-2012-10819.

\section{References}

[1] N. Tantalo, "Isospin Breaking Effects on the Lattice," PoS LATTICE 2013 (2014) 007, doi:10.22323/1.187.0007 [arXiv:1311.2797 [hep-lat]].

[2] FLAG, S. Aoki and others, Eur. Phys. J. C77 (2017) 2, doi:10.1140/epjc/s10052-016-4509-7 [arXiv:1607.00299 [hep-lat]].

[3] M. Heffernan, P. Banerjee and A. Walker-Loud, "Quantifying the sensitivity of Big Bang Nucleosynthesis to isospin breaking with input from lattice QCD," arXiv:1706.04991 [nucl-th].

[4] A. Bussone, M. Della Morte and T. Janowski, "Electromagnetic corrections to the hadronic vacuum polarization of the photon within $\mathrm{QED}_{\mathrm{L}}$ and $\mathrm{QED}_{\mathrm{M}}$," doi:10.1051/epjconf/201817506005 [arXiv:1710.06024 [hep-lat]].

[5] M. Tanabashi et al. (Particle Data Group), Phys. Rev. D 98, 030001 (2018), doi:10.1103/PhysRevD.98.030001.

[6] J. Bijnens and J. Prades, "Electromagnetic corrections for pions and kaons: Masses and polarizabilities,” Nucl. Phys. B 490 (1997) 239, doi:10.1016/S0550-3213(97)00107-7 [hep-ph/9610360].

[7] J. Bijnens and N. Danielsson, "Electromagnetic Corrections in Partially Quenched Chiral Perturbation Theory," Phys. Rev. D 75 (2007) 014505, doi:10.1103/PhysRevD.75.014505 [hep-lat/0610127].

[8] S. Basak et al. [MILC Collaboration], "Lattice computation of the electromagnetic contributions to kaon and pion masses," arXiv:1807.05556 [hep-lat].

[9] S. Borsanyi et al., "Ab initio calculation of the neutron-proton mass difference," Science 347 (2015) 1452, doi:10.1126/science.1257050 [arXiv:1406.4088 [hep-lat]].

[10] F. B. Erben, P. E. Shanahan, A. W. Thomas and R. D. Young, "Dispersive estimate of the electromagnetic charge symmetry violation in the octet baryon masses," Phys. Rev. C 90 (2014) no.6, 065205, doi:10.1103/PhysRevC.90.065205 [arXiv:1408.6628 [nucl-th]].

[11] F. Sanfilipo, C. Tarantino, G. Martinelli, C. Sachrajda, S. Simula, N. Tantalo, D. Giusti, V. Lubicz, "Radiative corrections to decay amplitudes in lattice QCD," Lattice 2018. 